\title{
Das Passiv im Deutschen
}

Akten des Kolloquiums

über das Passiv im Deutschen,

Nizza 1986

Herausgegeben vom

Centre de Recherche

en Linguistique Germanique (Nice)

Sonderdruck

Max Niemeyer Verlag Tübingen 1987 
Z U M P A S S I V I M A L T H O G H D E U T S C H E N I S I D O R.

Versuch einer valenzsyntaktischen Beschreibung.

Ludwig M.Eichinger

1. VON DER VERWANDTSCHAFT DER GENERA VERBI.

Uber lange Zeit schienen die Abstammungs- und Verwandtschaftsverhältnisse innerhalb der Familie GENERA VERBI gänzlich unproblematisch zu sein. Das Passiv galt seit undenklichen Zeiten als - um im Bilde zu bleiben - leiblicher Abkomme des Vaters Aktiv, weniger metaphorisch: das Passiv galt als eine Konverse, die sich auf relativ einfache Weise vom entsprechenden Aktivsatz ableiten ließe. Innerhalb der längst sanft verschiedenen standardvariante der gTG galt diese in Transformationsregeln gegossene Weisheit als Beleg für die Tatsache, daß auf solche Weise etwas zu erkennen wäre, was andrenfalls für immer unentdeckt bleiben müBte. Wie Harald weinrich in der Erben-Festschrift unlängs ausgeführt hat, läßt sich aber sowohl diese Analyse weit, genauer bis auf die aristotelische Kategorienlehre zurllckfuhren - Weinrich nimmt das zum Anlaß, überhaupt von der aristotelischen Analyse zu sprechen - außerdem ist auch die generative Produktionsmetaphorik kaum jünger. ${ }^{1}$

Dieser Beitrag Weinrichs gehört zur Spitze eines Eisbergs: in den letzten Jahren mehren slch unübersehbar die Kritiker. der aristotelischen Analyse, nicht zuletzt ist im derzeitigen Modell der generativen Transformationsgrammatik dieser Ableitungsweg aufgegeben. So geht man nun in der Regel davon aus, daß Aktiv und Passiv zwei nicht voneinander ableitbare, aber miteinander verwandte Konstruktlonsweisen sind.2

Wenn ich Weinrich zudem recht verstehe, sieht er somit auch nicht allzuviel Sinn darin, Passiv uber elne morphosyntaktische Umformulierung allein zu beschreiben, sondern von einer lexikalisch bedingten Reduktion der Argumente auszugehen. Damit ergibt sich aber zwangsläufig, daß die Art und Möglichkeit solcher Argumentreduktionen nur innerhalb einer Beschreibung sinnvoll diskutiert werden kann, die die Muster von Handlungsrollen, die semantisch gegliederte Verbgruppen um sich aufbauen in Aktiv, der Verteilung derselben Handlungsrollen im Passiv

1 S. Weinrich (1985: 357\&359f.).

2 S. z.B. Eiseņberg (1986: 133). 
130

gegenüberstellt. Erfreulicherweise schließt auch weinrich (1985: 360): "Mir scheint, daß eine adäquate Theorie der Verbal-Genera Aktiv und Passiv (...) heutigentags nur im Rahmen einer wohlgeformten Valenztheorie gegeben werden kann."

Wir ziehen aus solchen Uberlegungen den Schluß, daß man für die Erklärung der strukturbildenden wirkung des Passivs nicht von einer einfachen Umdrehung der in Aktiv realislerten Relationen ausgehen darf, wohl aber von einer Verwandtschaft vor Aktiv und Passiv in dem Sinn, daß das Aktiv - in sogenannten AktivSprachen zumindest - die unmarkierteste und vollständigste Konstellation der Handlungsrollen elnes Verbs bzw. einer Verbgruppe repräsentiert. Auch die Art der Anbindung der einzelnen Elemente, d.h, die Korrelation von semantischen Strukturen und grammatischen Formen hat im Aktlv als die unmarkierte Variante zu gelten.

Eine Beschrejbung, die diesen hier grob skizzierten Faktoren Rechnung trägt hat also (1) die Konstellation der Handlungsrollen für das jeweils betrachtete Verb zu beschreiben, (2) die syntaktisch-hierarchische Umsetzung der Rollenkonstellatlonen in den verschiedenen GENERA VERBI, dazu möglicherweise (3) selektionale Sonderbedingungen zu beachten, Jetztlich (4) die Umsetzung in syntaktische Valenzen anzugeben.

\section{PASSIV IM ALTHOCHDEUTSCHEN ISIDOR}

\subsection{Das Vorhaben}

Es soll im folgenden am Beispiel einiger Verben und ihrer Belege aus dem althochdeutschen Isidor versucht werden, nachzuweisen, daß solcherart einerseits die Verhältnisse zwischen Aktiv und Passiv beschreibbar sind, und zusätzlich in einer Weise, die Aussagen über den Status der verschledenen Konstruktionstypen zuläßt. ${ }^{3}$

3 Zu einer genaueren Beschreibung des benutzten syntaktischen Modells sieh Eichinger (1985) und Eichinger (1987), im letztgenannten Titel werden auch die einschlägigen Arbeiten zur historischen Anwendung der Valenzgrammatik wie zu den behandelten ahd. Problemen ausfuhrlich besprochen. Im folgenden wird versucht, den Unterschied der behandelten Formen als tendenziell grammatikalisierte Möglichkeiten zur unterschiedlichen Anordnung der durch das Verb aufgespannten Szenen $z u$ verstehen und $z u$ beschreiben, u.d.h. mit satzsemantisch-lexikalischen, syntaktischen und textuellen Mitteln. Es wird der Anspruch erhoben, daß damit ein entscheidender Faktor für den Unterschied wischen den verschiedenen genera verbi erfaßt wird. Das schließt nicht aus, daß es weitere, nicht unbedingt kongruente Funktionen dieser grammatischen Instrumente gibt. 
Der Bezug auf elnen althochdeutschen Text hat hierbel den Vorteil, daß er mehr Schwièrigkeiten macht. Das ist in zweierlei Hinsicht ein Vorteil: (1) ist so der bel neuhochdeutschen Texten ungebrochene Rekurs auf ein naives Ohnehin-schonWissen dessen, was man beschreiben will, ganz deutlich gebrochen, und zum zweiten 1st ja die Frage, wie es um die Existenz und Funktion der verschiedenen PASSIV genannten Konstruktionsweisen im Althochdeutschen überhaupt steht, nicht eindeutig geklärt.4

Beide Arten von Schwlerigkeiten konvergieren praktisch zu dem Problem, wie man möglichst zweifelsfrei die Korrelation von grammatischer Konstruktion und Verteilung der Handlungsrollen für einen bestimnten Verbbeleg des Althochdeutschen möglichst unstrittig ermitteln kann. Denn wenn stimmt, was Hans-Jürgen Heringer bildhaft so umschrieben hat: "Ein verb, das ist so, wie wenn man im dunklen Raum das Licht anknipst. Mit einem Schlag ist dle Szene da."(1984: 49), dann haben wir das Problem. wie sich der althochdeutsche Textproduzent die Szene vorstellte, die er mit der Lampe des jeweiligen verbs aus dem Dunkel zu holen gedachte. Da häufiger so getan wird, als handle es sich hierbel um ein spezielles Problem der valenzgrammatischen Beschreibung, sei darauf verwiesen, daß die klärung der Erage nach der Funktion ja der Existenz bestimmter Aktiv- und Passivkonstruktionen im Althochdeutschen auf jeden Fall erfordert, die entsprechenden Bedeutungsunterschiede aus den sprachlichen Fakten zu rekonstruieren. Ohne $\mathrm{Hy}-$ pothese über die Fakten kann man also auf keinen Fall auskommen.

\subsection{Die Beispiele}

Was damit gemeint ist, sei nun am Beispiel der Belege einiger Verben des ZEIGENS und AUSSERNS, wie man sie vorläufig zusammenfassend nennen könnte, die sich im ahd. Isidor finden, dargelegt. Es soll dabei um die Verben araugen vor Augen stellen', meinan 'bezeichnen, meinen' und quhedan 'reden' gehen. Aus Gründen der leichteren methodischen Kontrollierbarkeit - d.h. um sonstlge Variation auszuschalten - beziehe ich mich dabei strikt auf den Text des Pariser Codex, whe er in der Ausgabe von G.A.HENCH aus dem Jahre 1893 vorliegt.

\subsection{1. araugen}

In dem genannten Text ist das Verb araugen $17 \mathrm{mal}$ belegt, davon $7 \mathrm{mal}$ in einer einfachen flniten Verbform, einmal als Partizip II in Verbindung mit einer fi-

4 Vgl. dazu die ausführliche Diskussion in Schrŏder (1955) und

Rupp (1956); auch die entsprechenden Ausführungen in

Sonderegger (1979: 272ff.) und Wolf (1981: 80ff\&204f.). 
niten Form von werdhan 'werden' und die restlichen 9 Male als Partizip II in Verbindung mit einer finiten Form von wesam/siln 'sein`. In der folgenden Liste werden für die Belegtypen EINFACHES PRADIKAT (=1.1.) und PART II + WERDH- (=1.2.) je ein, für den häufigsten Typ PART II + WES- (=1.3.) zwei Belege gegeben:J

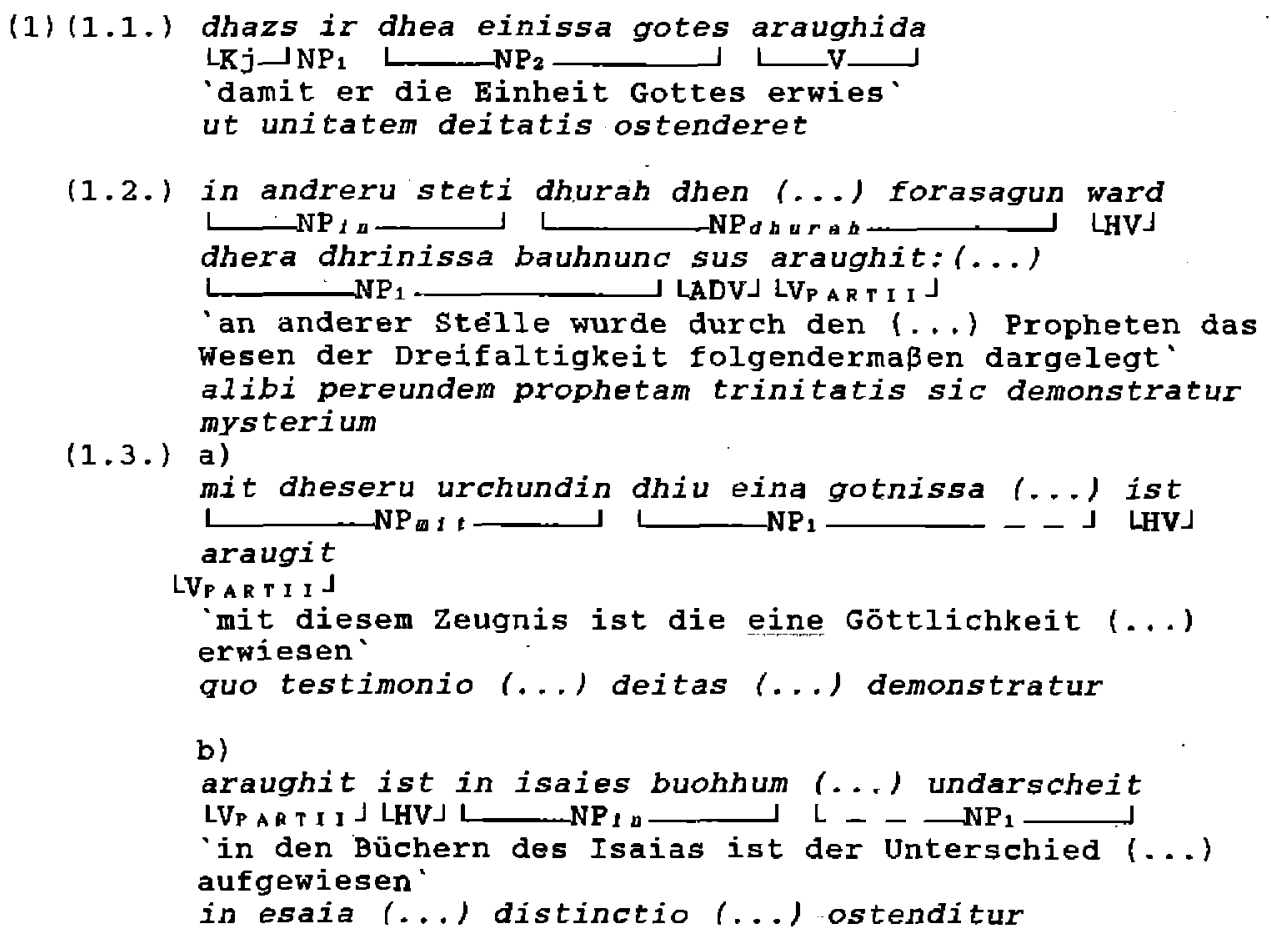

Dabel sei Im folgenden kürzer auf die AKTIV- und VORGANGSPASSIV- Konstruktionen, stärker auf das ZUSTANDSPASSIV, wenn ihre vorläufige Benennung so erlaubt sei, eingegangen.

5 Im weiteren werden die folgenden abkurzungen benutzt:

$S=$ Satz (in Băumen absteigend durchnumeriert $S_{i}$ usw.

$\mathrm{V}=\operatorname{Verb}\left\langle\mathrm{V}_{2}=\mathrm{z}\right.$ weiwertiges, $\mathrm{V}_{3}=$ dreiwertiges $\mathrm{V}$;

$\mathrm{HV}=$ Hilfsverb ggf. auch $V_{15}=V$ mit $E_{1}$ und $E_{5}$ [s.u.])

NP $=$ Nominalphrase

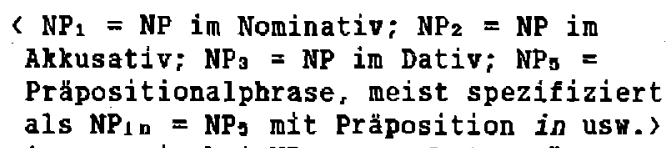


Als formale Valenz ergibt sich filr alle Aktiv-Belege Zweiwertigkeit mit Fnow und Esk. Von den vorkommenden adverbialen Bestimunngen ist keine regelmäßig genug vertreten, als daß Ihr Status als Ergänzung oder Angabe diskutiert werden mußßte: es handelt sich durchwegs un Angaben. Allerdings ist eine der denkbaren adverbialen Bestlmmungen durch die subjektwahl in den vorliegenden Sätzen ausgezelchnet. Während nämlich in unseren Aktiv-Belegen als Eakk durchweg die Tatsache, die dargestellt oder bewiesen wird, genannt wird, tritt als Enow neben dem Agens der Handlung das Instrument des Beweises bzw. das Mittel der Darstellung auf. Das ließe elnen Bezug auf folgendes Muster von Handlungsrollen zu:

(2) Jmd. (AGENS) stellt dar/beweist die Tatsache, daß... (OBJEKT), indem er dazu die (andere) Tatsache benutzt, daß... (INSTRUMENT)

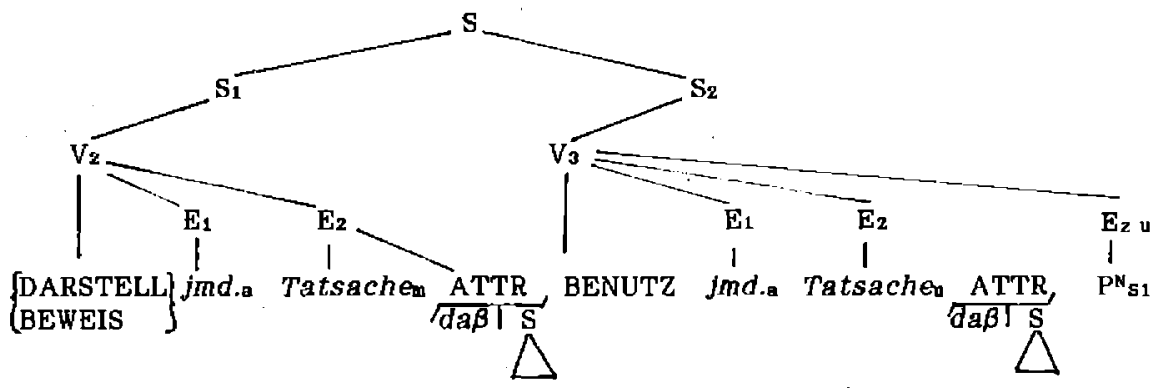

Es sei hier bemerkt, daß es sich hierbei um eine sprachllche "gestreckte" Ausformulierung der Verhåtnisse handelt, die sich aus dem Gebrauch der beteiligten Elemente in den einzelnen Belegen ablesen läßt. ${ }^{6}$ Die Vertellung der hier vorgegebenen Rollen auf Satzglieder, d.h. die Umsetzung in eine mehr oder minder hierarchisierte syntaktische struktur kann als eine über eine Art lexikalisierter Thematisierungsoperationen gesteuerte Umformung verstanden werden. Konversen wie das Passiv stellen dann Möglichkeiten zu einer in bestimmter Weise markierten Thematisierungsmöglichkeiten innerhalb des durch das verbale Lexem vorgegebenen Rahmens dar. Die damit angenommene Ableitung von Aktiv und Passiv von derselben Basls soll darstellen, da $\beta$ zwischen diesen Konstruktionstypen Familienähnlichkeit besteht, aber keine direkte Deszendenz anzunehmen sind. Allerdings ist für den Sprachtyp des Deutschen die Art von Thematisierung, die zum Passiv führt, als marklerter anzusehen, als die Normalthematisierung des Aktivs, wie sie sich etwa aus den Lexikoneinträgen des jeweiligen Verbs entnehmen läßt.

6 Zur Symbolisierung vgl. Eroms (1981) und Eichinger (1985). 
Für das Aktiv ist soweit aber entsprechend den Belegen nur beschrleben, daß Konstruktionen wie in Beleg (1.1.), mit der handelnden Person als Subjekt, und solche mit dem Mittel oder Instrument als Subjekt wie z.B.

\section{(1.1.)(1) Dhiu selba maneghlu chinomidiu araughit chirunl dhera dhrinjssa \\ 'ebendiese Vielzahl der Personen belegt das gewaltige Geheimnis der Dreifaltigkeit'.}

Auch das Vorgangspassiv - einziger Beleg mit diesem Verb in unserem Text ist der oben unter (1.2.) belegte - läßt sich auf die oben angedeutete Baslsstruktur beziehen, und zwar, konkret aufgefüllt, etwa in folgender Weise:

(3) der Prophet stellt die Tatsache, wie die Drelfaltigkeit ist, dar, indem er dazu 〈folgende〉 Darstellungwelse benutzt

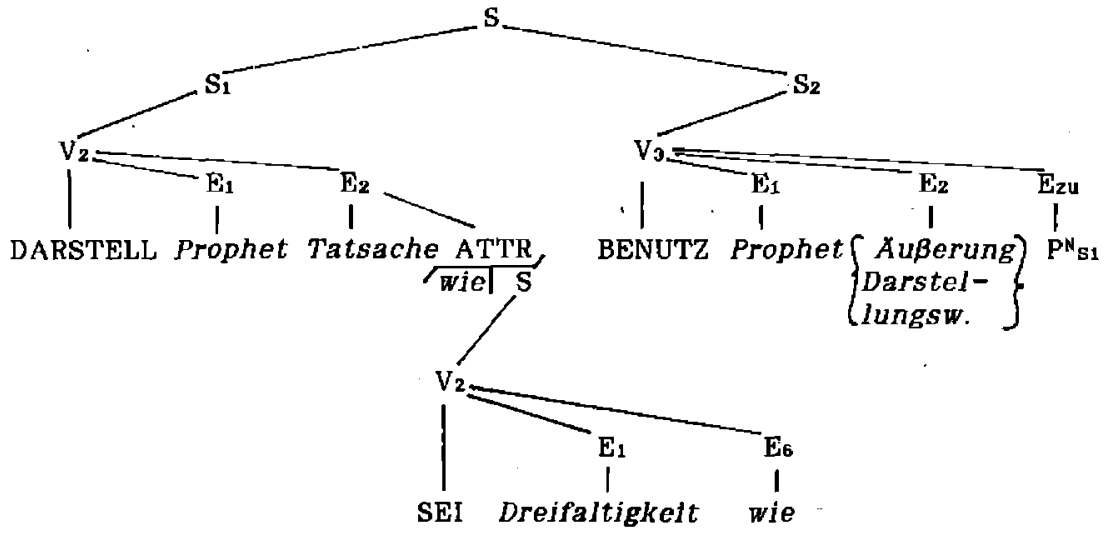

Auf der Ebene der syntaktischen Hierarchisierung Ist dann eindeutig zu erkennen, daß direkt eindeutig die Tatsache, die bei einem denkbaren Aktivsatz als $N_{z}$ auftauchen würde, als Subjekt gewählt würde, in diesem Sinn eine Valenzminderung gegenüber der Auswahl des Aktlvs bzw. dem gesamten Handlungsrollenkonzept des Verbs anzunehmen ist, wo ja zwei Ergänzungen kasuell an das Verb gebunden, somit zweifelsfrei davon regiert werden. In unserem Beleg wird nun zweifellos die für das Vorgangspassiv immerhin noch mögliche Variante gewählt, den Agens - allerdings, wie der auch leicht wechselnde prăpositionale Anschluß zeigt zusätzlich - in der dhurah Phrase noch anzugeben. Schon die Schwierigkeit, in gegenwartssprachllchen Analysen diesem Element eindeutig Ergänzungs- oder Angabencharakter zuzuweisen, zeigt allerdings, daß es sich um einen sekundären Einbezug dieser Relation handelt. Zudem ist der Schluß, diese Fügung müsse der Agens sein, nicht zwingend, es könnte durchaus sein, daß es sich hlerbei lediglich um eine instrumentale Anfügung handelt, wenn in dieseln Satz gesagt werden soll, daß 
der weiterhin ungenannte Agens sich des Propheten als eines Instruments bedient hätte; das würde dann etwa zu folgender Interpretation führen:

(3) a) ' 'der ungenannte Agens (=Gott?)〉 stellt dar, wie die Dreifaltigkeit ist, indem er den Propheten benutzt, der äußert: (...)

Insofern man nun im Sinne des Texts davon ausgehen kann, daß Tatsachen, die implizit auf Kundgebungen Gottes zurückgeführt werden können, einfach Wahrheiten sind, ließe sich auch ibberlegen, ob das hier repräsentlerte Relationenkonzept diese Instanz überhaupt noch enthălt, oder ob araugit werdhan eher einem in dieser Hinsicht neutralen Prädikat wie KLARWERD entspricht:

(3) b) 'daß man den Propheten benutzt, der äußert:..., hat zur Folge, daß klar wird, wie die Dreifaltigkeit ist.'

Die relative Hăufigkeit, mit der allerdings generell beim sogenannten Vorgangspassiv noch der Agens hinzugefügt wird, mag eine so weitgehende Abtrennung auch in den Basisrelationen vielleicht noch nicht ratsam erscheinen lassen. Auch so ist auf jeden Fall der Agens aus der engeren Handlungsrollenauswahl ausgegrenzt, die Valenz an dieser Stelle gemindert, wie auch immer man das beschreiben mag.

Einen deutlichen Schritt darüber hinaus erlauben uns allerdings die neun Belege mit unserem Verb, die als Zustandspassive verstanden werden können. Die Perfektivität dieses Konstruktionsmusters hat Folgen in der Rollenauswahl, die sich auch in der syntaktischen Struktur weitaus deutlicher zeigen. Was liegt inhaltich in all den Zustandspassivbelegen vor? Es wird eine Tatsache als ein Zustand beschrieben, der auf eine Zeige-, Darstellens- oder Beweisens-Handlung zurückzuführen ist. Wenn. man dabei zur Verdeutlichung auf den lateinischen Paralleltext zurückgreift, so wäre patet 'es ist offenkundig' das Idealvorbild, insofern es den Zustand ausdrückt, allerdings ist dabei die Fundiertheit dieses zustands in einer Zeigehandlung nicht im selben Ausmaße vorgegeben. In den anderen lateinischen Parallelen - vom Typ ostenditur - sind dafür Vorgangs- und Zustandsausdruck formal nicht unterscheidbar. Der althochdeutsche obersetzer des isidorischen Traktats hat aber nun offenbar in seinem muttersprachlichen Inventar eine Konstruktion, die beides leistet, nämlich Darstellung eines Zustands einerseits und andrerseits Darstellung dleses zustandes als eines, der aus einer Handlung hervorgegangen ist; das ist eben das Zustandspassiv. Direkter Einfluß aus dem lateinischen Vorlagetext ist dabel, wie an den Parallelformen zu sehen, nicht anzunehmen. Denn im lateinischen T'ext wird formal gerade nicht so getrennt. Daß aber unser übersetzer eine solche Trennung Intendiert, wird durch seine differenzierte oberset- 
zungsstrategie nahegelegt: so wird, wie auch aus unseren Belegen unter (1) zu ersehen ist, lateinisches demonstratur je nach Zusammenhang mit Vorgangs- oder Zustandspassivform wiedergegeben, andrerseits werden sowohl manifestatur, ostenditur, demonstratur wie andrerseits auch patet mit Zustandspassiv übersetzt. Auch vulgăr- bzw. mittellateinischer Einfluß schelnt an dieser stelle nicht besonders naheliegend, da sich dort die esse-Paraphrase als Ausdrucksmittel für das Vorgangspassiv installiert. Vor dlesem Hintergrund ware die Art der Differenzlerung im althochdeutschen Isidor - v.a die Entstehung der werdhar-Paraphrase für das Vorgangspassiv - etwas iberraschend. Wesentlich einleuchtender erschiene es mir, hier Lehneinfluß in dem Sinne anzusetzen, daß gerade das Bewußtwerden der Differenzen in der Ausdrucksweise zum latelnischen Vorbild beim Ubersetzer die Differenzierung des deutschen Paradigmas beförderte.

Eine genauere Distributionsanalyse bestătlgt die Deutung der vorliegenden Konstruktion:

(4)

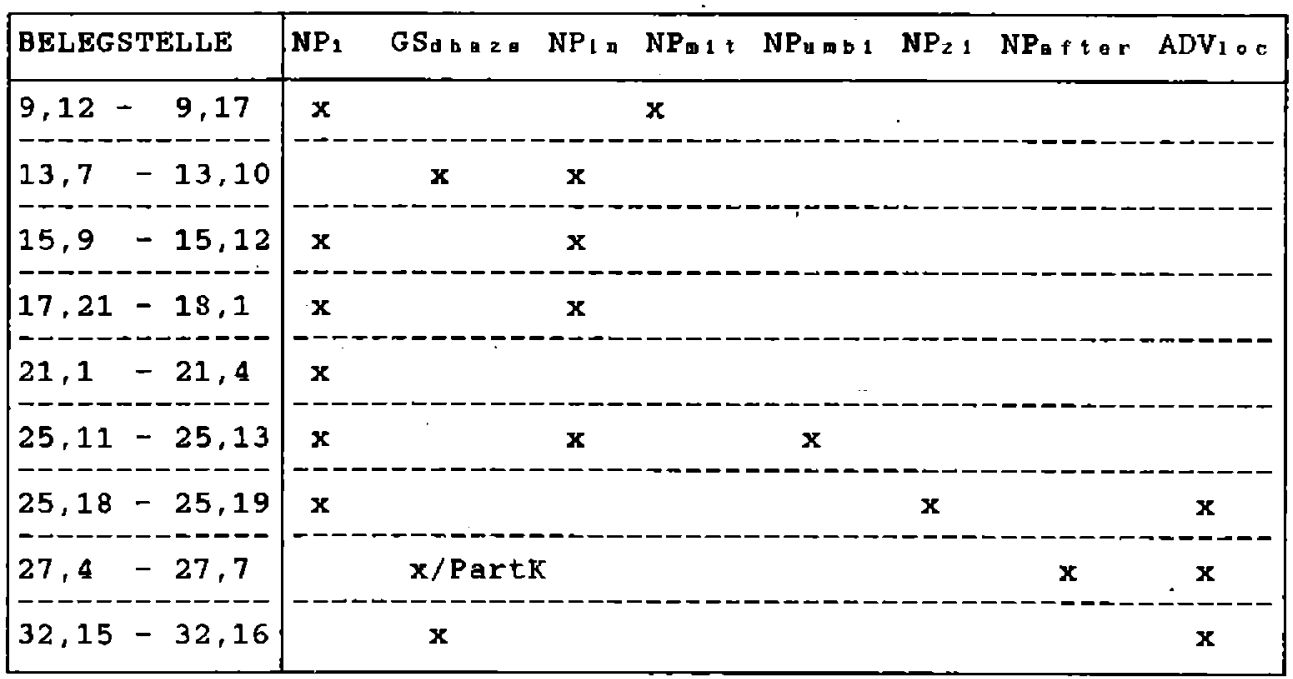

Wie auch ober beim Vorgangspassiv wird die Subjektsposition beim $\mathrm{Zu}-$ standspassiv von jenen Tatsachen besetzt, die beim Aktlv an der Stelle der Eakk. auftauchen. Diese Position ist hier durch nominativische Nominalgruppen (nur Flexion bel pronominalem Subjekt) oder Konjunktionalsätze mit der Konjunktion dhazs realisiert - ebenfalls entsprechend dem Aktivbefund. Kennzeichnender - auch im Hinblick auf die Absetzung vom Vorgangspassiv - ist aber ein anderer Punkt. Die 
in vier von den neuen Belegen vorhandenen NPin sind lokale Bestimmungen und somit semantisch mit den Lokaladverbien in drei weiteren Belegen zu einer adverbialen Position zusammenzufassen. Ein Beleg zeigt - stattdessen - eine instrumentale NPmlt. Beim verbleibenden Beleg - dem ersten aufgefuhrten - ist die entsprechende lokale Bestimmung dem Kontext zu entnehmen. Schon von den Zahlenverhältnissen her wäre somit - z.B. nach Greules Bestimmung sogenannter affinitativer Adverbialia $\rightarrow$ eine lokale Ergänzung für dieses Konstruktionsmuster anzunehmen. Erst die Deutung dieser Elemente in ihrer Funktion für das Verständnis erlaubt es dann jedoch, zum ersten diesen numerisch nahegelegten Befund zu begründen, und zum zweiten, auch die instrumentale Bestimmung in dlese Ergänzungsklasse mit einzubeziehen. Methodisch einigermaßen vertretbar formuliert heißt das, es sind Präpositlonalgruppen mit den Präpositionen in und mit und elner aufzählbaren Menge funktional entsprechender Adverbien anzusetzen. Aup der selektionalen Ebene ist dann zu sichern, daß nur Adverbien bzw. Präpositionen mit den entsprechenden lokalen oder instrumentalen Lesarten gewählt werden.

Wenn wir versuchen, diesen formalen Befund auf die in unserem Text vorliegenden inhaltlichen strukturen abzubilden, heißt das, daß an dleser stelle in den Sätzen die Begründungen für den Beweis der an Subjektstelle aufgeführten Tatsachen genannt werden. Als solche Begründungen gelten im Rahmen der vorliegenden Textsorte theologisches Traktat Außerungen in den heillgen schriften. Dabei können in den sabtzen sowohl die Außerungen selbst oder ihr Inhalt auftauchen: In diesem Fall sind sie Instrument des Beweises, entsprechend dem Anschluß mit mit. Es kann aber auch metonymisch auf die Bücher. In denen diese Außerungen zu finden sind, Bezug genommen werden: das führt zu den entsprechenden Bestimmungen mit in.

Zu dieser inhaltlichen Beschreibung des hier realisierten Satzmusters paßt auch, daß im Rahmen der intendierten Zustandsbeschreibung in keinem Beleg der Agens der Handlung, die zu dem Zustand geführt hat, genannt wird. Auch die Verwendung gerade einer solchen Konstruktion ist gut im Zusammenhang der vorliegenden Textsorte zu deuten. Die in den instrumentalen oder lokalen Bestimmungen genannten Erscheinungen sind entweder die blbllschen Außerungen selber oder die Bücher, in denen diese Außerungen stehen. Sie gelten, als von Gott gegeben, als autoritative Begriandung für dle zu beweisenden und in solchen sätzen als offenkundig gesetzten Glaubenstatsachen. Der Urheber. als solcher steht damit sprachlich iiberhaupt nicht mehr zur Debatte. Auch die lateinischen Parallelbelege sind durchweg agenslos. Damit geht es hier inhaltlich eher um den Tatbestand, daß etwas

7 S. Greule (1982: 194). 
sozusagen aufgrund bestimmter Außerungen mathematisch bewiesen ist, wobei die Urheberschaft Gottes axiomatisch gesetzt, aber nicht als im Einzelfall eingrelfend gedacht ist.

Aufgrund der formalen Befunde, die sich bei diesem Muster finden und aufgrund der gerade angestellten Inhaltlichen Oberlegungen würden wir hler dafür plädieren, daß es sich hier in Vergleich zu Aktivverwendungen nicht nur um eine Umthematisierung mit einer gewissen Valenzreduktion im Rahmen derselben sprachlichen Szene handelt, sondern um einen sprachllchen Szenenwechsel, in eine Szene mlt einem Zustandsverb, wobel nur mehr auf tieferer Ebene der Bezug auf die Zelgenshandlung erkennbar 1st. Für unseren Fall scheint mir das ein Prädikat wie HERyORGEHEN AUS zu seln. Die Relatlonen der Handlungsrollen lleßen sich bel dieser Annahme folgendermaßen formulieren:

(5) 'die Tatsache, daß... geht daraus hervor, daß Gott diese Tatsache darstellt/beweist, indem er dazu eine Außerung, die ein Autor in einem Buch gemacht hat, benutzt'

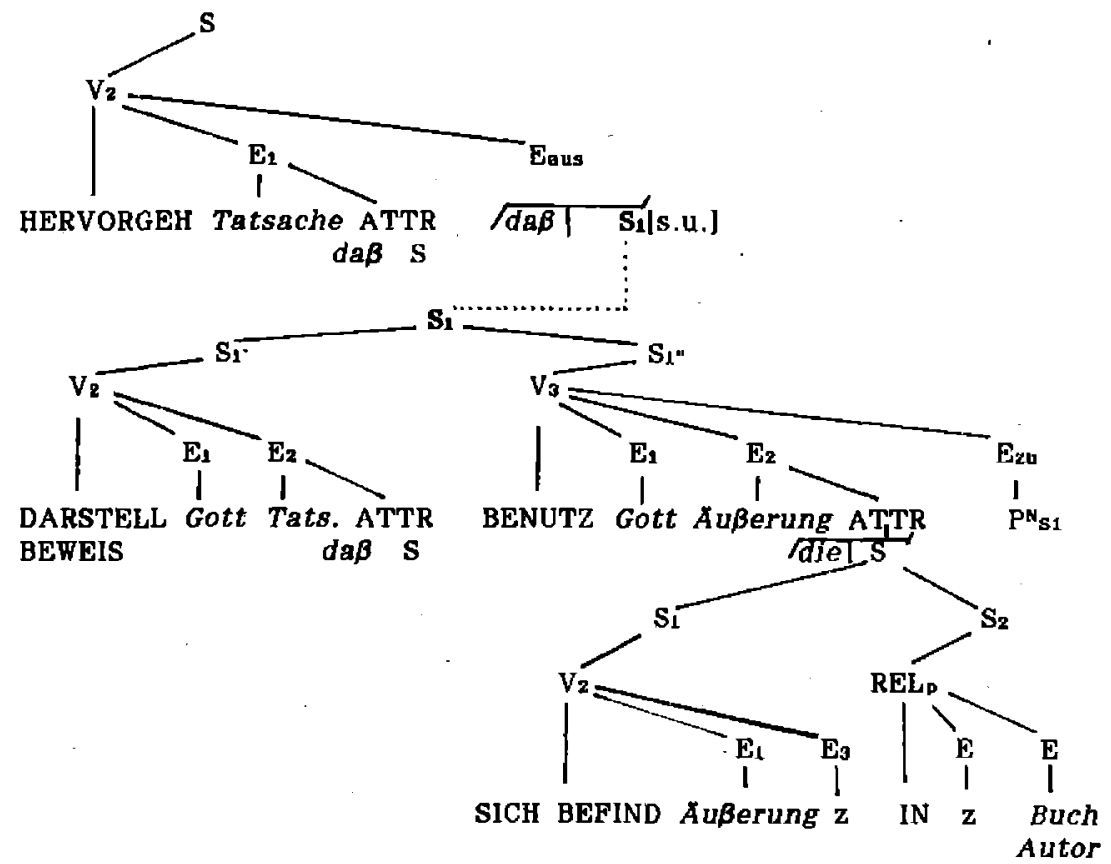

ggf. auch:

'eine Außerung. dle ein Autor ln einem Buch (der hl.Schrift) macht, hat zur Folge, daß dle Tatsache, daß... klar ist." 
Daraus läßt sich als minimale Form - valenzgemindert - ein elnwertiges $x$ Ist araughit ableiten, für unseren Text wird zudem nahegelegt, daß diese im Hinblick auf das aktive araugen valenzreduzierte arsught wessn durch den 0bertritt in das Muster von HERVORGEHEN AUS bestimmte Typen präpositionaler Ergănzungen zu sich nimmt, so daß das Zustandspassiv ein zweiwertiges komplexes Prădikat mit E 1 und Enit/1n/advdarstellt

(6)

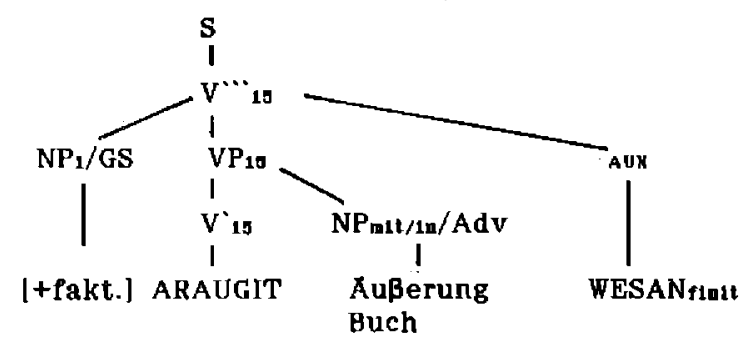

bzw.<smiles>[Z16][Y19]([R1])S</smiles>

Die hier skizzlerte, besonders auf die semantisch-kategoriale Rahmenstruktur und ihre sprachliche Fassung Wert legende Valenzanalyse macht es wahrscheinlich, daß es sich bel den behandelten Konstruktionsmustern zumindest tendenziell um die Ausdifferenzlerung eines Paradigmas Vorgang vs. Zustand In Bezug auf das objekt eines entsprechenden Aktivsatzes handelt. $\mathrm{Zu}$ dieser Paradigmatisierung werden analytische Formen mit den Hilfsverben wesan/siin bzw. werdhan geschaffen und genutzt. Unter dem Vorbehalt, daß blsher nur die verwendung eines verbalen Lexems bei einem Autor untersucht wurde, kann man damit konstatieren, daß unsere Beschrelbung, da sie sowohl Unterschiede wie Zusammenhănge zwischen den Konstruktionen sichtbar macht, den synchronen Befund in einen erklärenden Zusammenhang stellt.

\subsection{1. meinan}

Dle Im folgenden noch kurz besprochenen weiteren Verben sollen dazu dienen, die im ersten Fall vorgeschlagene Art der Analyse zu überprüfen. Ihre Anordnung hier erfolgt rein alphabetisch. 
Das Verb meinan ist in unserem Text an 11 stellen belegt; es handelt sich um 8 Belege als finites Aktiv-Verb und um 3 Belege mit PARTII von meinan und elner finiten Form des Hilfsverbs wesan/siln. Die Aktivbelege zeigen ein durchgehend zweiwertiges Verb, mit $E_{1}$ und $E_{2} / E_{s E}$, sowie einen auch inhaltlich etwas abweichenden Beleg mit $E_{1}$ und Eumb:

(7) Dhar dhu chihoris umbl dhen chisalbodon got meinan, dum enim gudfs deum unctum

Daneben fỉndet sich in zwel Belegen eine präpositionale Bestimmung mit in - wobei eine lokal und eine instrumental zu verstehen ist - und in einem eine mit mit. Somit wären typische Aktlv-Belege etwa:

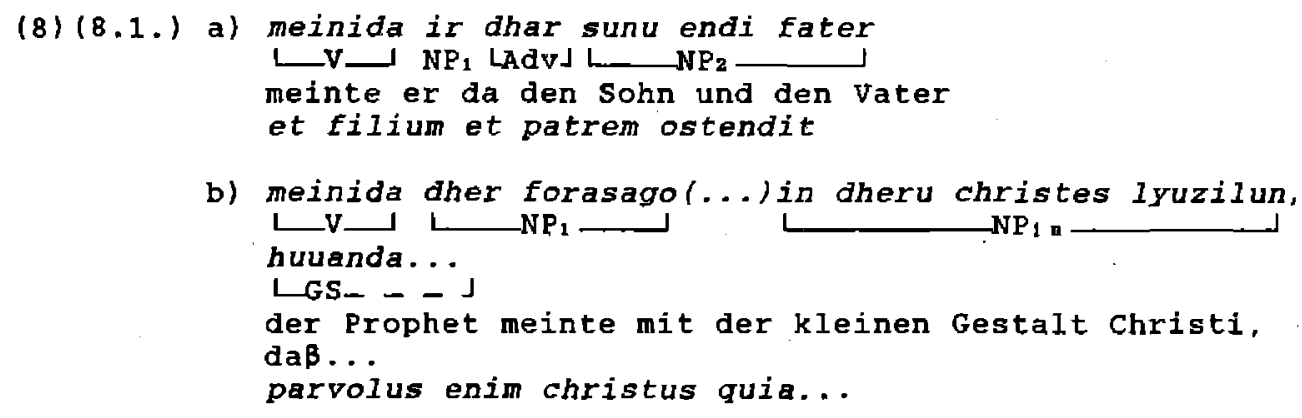

Auch hier zeigt sich, zudem, wenn man die textuellen Ungebungen der Belege mit berucksichtigt, eine bestimmte Wichtigkeit der lokalen und instrumentalen Bestimmungen, und zwar in derselben Weise wie oben, bestimmte Inhalte von biblischen Außerungen werden als Instrumente, mit denen etwas behauptet oder gemeint wird, eingefuhrt, bzw. die biblischen schrlften oder Teile von ihnen als die Orte, wo sich dlese AuBerungen finden. So wäre es wohl angebracht, etwa eine Basisstruktur whe die folgende anzunehmen:

(8)(8.2.) Jemand meint jemanden/etwas/eine Tatsache, indem er etwas, das sich in den hl.schriften befindet; äußert

D.h. diese prinziplell agentisch und auf ein objekt ausgerlchtete struktur zeigt eine analoge texttyplsche Besonderheit. wle sle sich auch bei araugen schon finden ließ.

Bei melnan nun finden sich, wje gesagt, kelne Fălle von Vorgangspassiv, dafür aber drei Fålle von Zustandspassiv; der typische Fall ist der folgende: 
(8)(8.3.) (...) ist christus in dheru (...) salbidhu chimeinit.

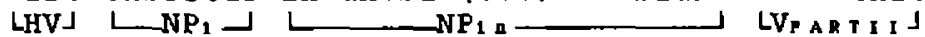

(...) ist Christus mit der $(\ldots)$ salbung gemeint

$(.$.$) christus (. .$.$) usctione monstratur$

Auch hier finden wir eindeutig wieder die Valenzreduktion, die zum normalen, sogenannten objektspassiv, führt. Ebenso wie oben wird elndeutig ein Zustand beschrieben, es wird nicht jemand gemeint, er ist auch nicht gemeint worden, sondern er ist in einem vorliegenden Bibelwort im Sinne des mehrfachen Schriftsinns "bedeutet". Dazu paßt, daß zwei der drei Belege eine NPin enthalten. die einmal s.o. - Instrumental, das andere Mal lokal zu verstehen ist: auch das Abhängigkeitsverhältnls zwischen diesen Bestimmungen läßt sich analog dem oben ausfïhrlich diskutierten Beispiel so sehen, daß der Ort der Außerung statt des Inhalts der Außerung stehen kann. In dem einen Beleg, wo keine solche satzsyntaktische Position gefüllt ist, wird sie anaphorisch Im Text aufgerufen. Auch die lateinischen Paralleltexte sind ähnlich wie im obigen Fall: einmal monstratur, einmal ostenditur und einmal ecce. D.h. auch hier keine perfektischen, sondern perfektive Vorbilder und eine typischerweise unagentische Konstruktionswelse. Infolgedessen solte auch die kategoriale Basis entsprechend unagentisch gewendet sein und auf die Bibel als Beweisgrund verweisen:

(9) Jemand/etwas ist gemeint, wozu eine Außerung, die sich in den heillgen Schriften befindet, dient'

Auch hier kommen wir also nach Valenzreduktion um den Agens über die systematische Umdeutung der Basis zu einer Konstruktion mit $E_{1}$ und En/mit bzw. entsprechenden Adverblen. Durch den Bezug auf zuständlich Vorliegendes wird die Zustandsbeschreibung in dem komplexen Prädikat aktualisiert.

\subsection{3. quhedan}

Mit diesem letzten Verb bewegen wir uns innerhalb der zentralen Gruppe von Außerungsverben, deren Unterschied sich ledlglich auf die Modalität der Außerung bezieht, so daß hier - bel Einbezug weiterer Verben - im wesentlichen parallele Ergebnisse zu erwarten wären.

Quhedan ist nicht nur das bei weitem meistbelegte Verb dieser Gruppe. sondern eines der häufigsten Im Isidor überhaupt. Insgesamt finden sich 68 aktivische und 9 passivische Belege, wobei bei den Passiven 5 Zustandspassivformen 4 Vorgangspassivformen gegenüberstehen. Bei den Aktiubelegen gibt es im wesentlichen ein Valenzmuster, das Verb wird zweiwertig konstrulert: bei den Ergánzungsklassen handelt es sich um $\mathbf{E}_{1}$ und EGs. Der inhaltliche Zusammenhang ist 
ganz klar: die Ei benennt den, der die Außerung macht, der Gliedsatz benennt das, was geäußert wird. Dabei handelt es sich ganz überwiegend um direkte Reden, also, wenn man so will, um GS: Auffăllig ist einer gewisse Hăfung von NPumbi/fona;eine solche Bestimulung tritt immerhin in 18 Belegen auf; in ihr wird der Bezugsbereich der zitierten Außerung zusatzlich benannt. Eine gewisse Häufigkeit zelgen daneben noch bestimmungen NP1n, dle ebenfalls nach bereits bekanntem Muster die Zitierstelle bezeichnen. Es geht dabei noch um 7 Belege. Weitere Bestimmungen kommen nur ganz vereinzelt vor, und brauchen hier nicht welter zu interessieren. Typische Beispiele wären also:

(10) (10.1.)

a) ir quhad: "christes chiburt..."

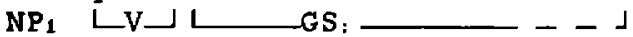

er sagte: "Christi Geburt..."

qui dicit: "generationem eius..."

b) umbi dhen auh in andreru stedi quhidhit: "..."

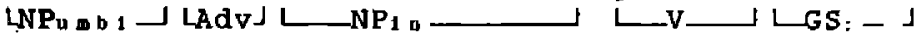

'von dem er auch an andrer stelle sagt:...

de quo et alibi in psalmis:...

Somit ergibt sich für die Aktiv-Belege eine Basisstruktur folgender Form:

(11) Jemand (AGENS) spricht etwas/eine Außerung (OBJ), die sich

in den helligen Schriften (LOC) befindet'

Bei diesem Verb nun llegen, wie wir sehen werden, Vorgangs- und Zustandspassiv sehr nahe beleinander. Für die drei normalen Vorgangspassivbelege der vierte sowie elner der Zustandspassivbelege gehören zu einer anderen Varlante von guhedhan, die uns hier nicht Interessieren soll - laßt sich folgende Distribution erkennen:

(12) $(12.1$.

dhemu in psalmom chiquedan ward

LNPa」 L NPI.n_L LVPA\&TII」 LHV」

von dem in den Psalmen gesagt wurde'

cui dicitur in psalmis

$(12.2$.

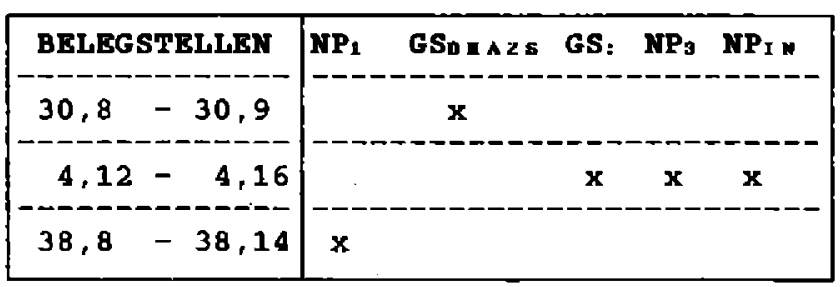


Ganz eindeutlg ist die Valenzreduktion erkennbar: die satzförmlge Ergänzung, dle die beweisende stelle aus den heiligen schriften direkt oder indirekt gibt, und die isn Aktiv. als objekt gewählt wird, tritt als subjekt und einzige Ergănzung auf; sie kann als konjunktionaler Nebensatz, als direktes Zltat oder als pronominal eln solches zitat wiederaufnehmende NP realisiert sein. In keinem Fall ist der Agens irgendwie genannt, stattdessen wird einmal der zitatort und die gemelnte Person genannt. Soweit wäre in diesem Fall auch die Ansetzung eines agenslosen Basismusters gerechtfertigt, wo auch die lateinischen Paralleltexte cul dicitur in psalmis:...; quod dictum est; llefern, lediglich der erste Beleg entspricht elner aktivisch gefaßten lateinischen Vorlage. Eine solche Basis könite lauten:

(13) 'es wird eine Außerung (OBJ) vorgelegt, die sich in den heiligen Schriften (LOC) befindet"

Auch hier könnte man allerdings in Analogle zu anderen Vorgangspassiven davon ausgehen, daß dle sekundäre Agensmarkierung potentiell möglich sein sollte. so daß eine Basis wie belin Aktiv anzusetzen wäre. Es zeigt sich aber, daß unsere Belege hier nurmehr Vorgang und Zustand bel ansonsten gleicher Basis zu differenzieren scheinen.

Das zeigt eine Betrachtung der Zustandspassivformen, dle folgendes Distributionsmuster ergeben:

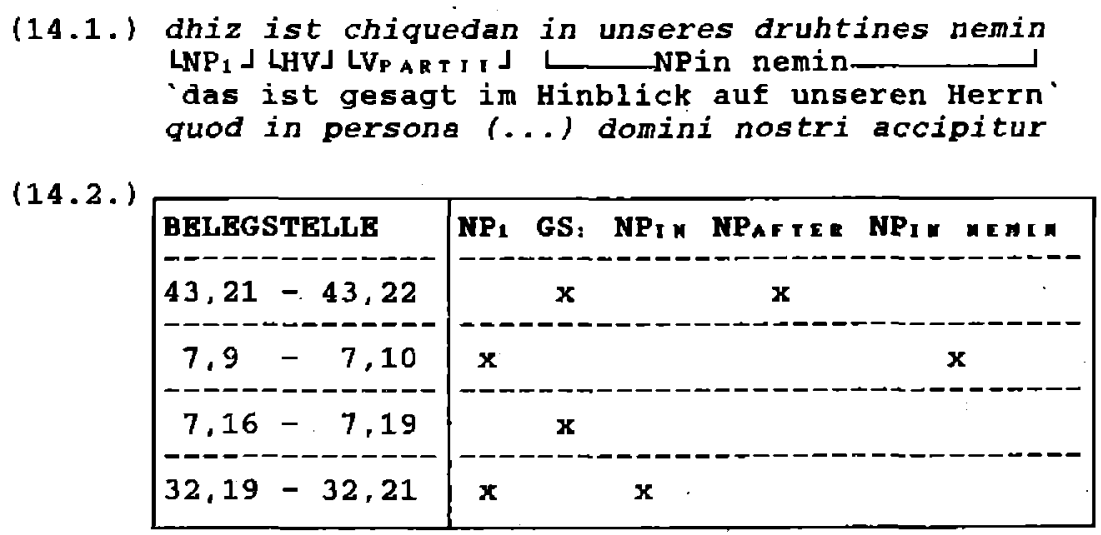

Auch hier haben wir es mit einer elnwertigen varlante zu tun, für deren Subjekt dieselben Bedingungen wie beim Vorgangspassiv gelten. Allerdings scheint hier durch die zustandsbeschreibung der Bezug aup elnen Agens prinzlplell ausgeschlossen; nur bestimmte lokale und modale Modifikationen werden angeschlossen. So wird man hier auf jeden Fall wiederum auf eine Modiflaktion des Befindllchkeitmusters zuriuckgreifen: 
(15) eine Außerung (OBJ) befindet sich in den heiligen Schriften (LOC), wobei jemand diese Außerung gesprochen hat (MOD/ CAUS)

3. SCHLUß

Die Untersuchung einiger Verben in althochdeutschen Isidor hat ergeben, daß über die Verteilung der Handlungsrollen elne Familienähnlichkeit zwischen den AKTIV, VORGANGSPASSIV und ZUSTANDSPASSIV genannten Formen hergestellt werden kann. Sie beruht in dem ähnlichen Inventar von Handlungsrollen, bestimmte Besonderheiten gleichen sich zudem aufgrund der Textsorte. Dle Blldung der Passivformen läßt sich als eine Art der Valenzminderung verstehen, bei der die an Subjektsposition auftretenden semantischen Rollen zunächst in eine periphere syntaktische Position gebracht werden, beim Zustandspassiv dann überhaupt aus dem Inventar möglicher Umgebungen ausgeschleden werden. Damit geht vor allem in diesem letzten Fall eine Umdeutung der Umgebungsbedingungen im Sinne bestimmter Zustandsverben einher, die eine weitere Verselbständigung der Zustandspassivkonstruktion zur Folge hat.

LITERATUR:

Abraham, Werner (Hg.), 1985. Erklärende Syntax des Deutschen. (Studien zur deutschen Grammatik 25) Tübingen. Narr.

Askedal, John Oie, 1985. Einige kritische Uberlegungen zur Unterscheidung von Ergänzungen und Angaben in der valenzlehre. Nouveaux cahiers d'Allemand 3. 113-21.

Dobnig-Jülch, Edeltraud, 1984. Zum Begriff 'komplexes Prädikat`. Studia Linguistica et Phllologica.' Festschrift für Klaus Matzel zum 60. Geburtstag überreicht von Schülern, Freunden und Kollegen, hg.v. Hans-Werner Eroms/Bernhard Gajek/Herbert Kolb. Heidelberg, Winter 353-65.

Ebert, Robert P. 1978. Historische Syntax des Deutschen. (SM 167) Stuttgart, Metzler.

Eggers, Hans, 1960. Vollständiges Lateinisch-Althochdeutsches wörterbuch zur glthochdeutschen Isidor-0bersetzung. (Deutsche Akademie der Wissenschaften zu Berlin. Veröffentlichungen des Instituts für deutsche Sprache und Literatur 20) Berlin, Akademie.

Eggers, Hans, 1964. Der althochdeutsche Isidor. Nach der Pariser Handschrift und den Monseer Fragmenten. (ATB 63) Tübingen, Niemeyer.

Eichinger, Ludwig M., I985. Die sprachliche Ausgestaltung von Raum und Zeit. Am Belsplel der verbalen Wortbildung in heutigen Deutsch. Habilmasch, Bayreuth.

Eichinger, Ludwig M., 1987. Zur syntaktischen Beschreibung fraherer Sprachstufen. Eine Fallstudie zum althochdeutschen Isidor. Althochdeutsch. Hg.von Rolf Bergmann u.a. Heidelberg, Winter

Eisenberg. Peter, 1986. Grundriß der deutschen Grammatik. Stuttgart, Metzler.

Eroms, Hans-Werner, 1981. Valenz Kasus und Präpositlonen. Untersuchungen zur Syntax und Semantik präpositionaler Konstruktionen in der deutschen Gegenwartssprache. (Monographien zur Sprachwissenschaft 11) Heidelberg. Winter.

Greule, Albrecht, 1982. Valenz, Satz und Text. Syntaktische Untersuchungen zum Evangelienbuch otfrlds von Weißenburg auf der Grundlage des codex Vindobonensis. München, Fink. 
Greule, Albrecht (Hg.), 1982. Valenztheorje und historische Sprachwissenschaft. (Reihe Germanistische Linguistik 42) Tübingen, Niemeyer.

Hench, George A., 1890. The Monsee Fragments. Newly collated text with introduction, Notes, grammatical Treatise and exhaustive Glossary and a photo-lithographic Fac-simile. Straßburg, Trübner.

Hench, George A., 1893. Der althochdeutsche Isidor. Facsimile-Ausgabe des Pariser Codex nebst critischem Texte der Pariser und Monseer Bruchstücke. Mit Einleitung, grammatischer Darstellung und einem ausfiuhrlichen Glossar. Straßburg, Trübner.

Heringer, Hans-Jürgen, 1984. Neues von der Verbszene. Pragmatik in der Grammatik. (Jahrbuch 1983 des IdS) hg. von Gerhard Stickel. Düsseldorf, Schwann. $34-64$

Lenerz, Jürgen, 1984. Syntaktischer Wandel und Grammatiktheorie. Eine Untersuchung an Beisplelen aus der Sprachgeschichte des Deutschen. (Linguistische Arbeiten 141) Tübingen, Niemeyer.

Matzel, Klaus, 1970. Untersuchungen zur Verfasserschaft, Sprache und Herkunft der althochdeutschen Obersetzungen der Isidor-Sippe. (Rheinisches Archiv 75) Bonn, Röhrscheid.

Plant, Helmut R., 1965. Zur Bestimmung von Wortart und Satzglied in althochdeutscher Prosa. Orbis 14. 487-95.

Plant, Helmut R., 1969. Syntaktische Studien zu den Monseer Fragmenten. (Janua Linguarum Series Practica 75) The Hague/Paris, Mouton.

Rupp, Heinz, 1956. Zum "Passiv" Im Althochdeutschen. PBB(H) 78. 265-86.

Schröder, Werner, 1955. Zur Passivbildung im Althochdeutschen. PBB(H) 77, 1-76.

Sonderegger, Stefan, 1979.: Grundzüge deutscher Sprachgeschichte. Bd.1. Einführung - Genealogie - Konstanten. Berlin, New York, de Gruyter.

Welnrich, Harald, 1985. Für eine nichtaristotelische Theorie der Aktiv-Passiv-Diathese in der deutschen Sprache. Studien zur deutschen Grammatik. Festschrift für Johannes Erben (...l hg. von Erwin Koller und Hans Moser. Innsbruck, Institut Pür Sprachwissenschaft der Universität, 357-74.

Wolf, Norbert Richard, 1981. Althochdeutsch - Mittelhochdeutsch. (Geschlchte der deutschen Sprache 1) Heidelberg, Quelle \& Meyer. 